\title{
Rapid detection of tert-butoxycarbonyl-methamphetamine by direct analysis in real time time-of-flight mass spectrometry
}

\author{
Ken-ichi Sugie ${ }^{1}$ (1) $\cdot$ Daisuke Kurakami $^{2} \cdot$ Mamoru Akutsu $^{1} \cdot$ Koichi Saito $^{3}$
}

Received: 1 November 2017 / Accepted: 15 December 2017 / Published online: 12 January 2018

(c) The Author(s) 2018. This article is an open access publication

\begin{abstract}
Purpose Phenethylamines constitute the majority of drug-related arrests in Japan. Recently, the smuggling of tert-butoxycarbonyl ( $t$-Boc)-protected phenethylamines has become of increasing concern, because of the difficult identification of these masked substances.

Methods In this study, a rapid and accurate method for the detection of $t$-Boc-methamphetamine ( $t$-Boc-MP) by direct analysis in real time-time-of-flight-mass spectrometry (DART-TOF-MS) was developed. The efficiency of the method was evaluated by comparison with conventional gas chromatography-MS (GC-MS) and liquid chromatography-TOF-MS (LC-TOF-MS) techniques.

Results During GC-MS analysis of $t$-Boc-MP, MP was generated in the injection port, which can lead to an analytical error. In the LC-TOF-MS spectrum, fragment ions were detected, which were generated by McLafferty rearrangement in the ion source. On the other hand, in the DART-TOF-MS analysis of $t$-Boc-MP, pyrolysis could be suppressed by using a microsyringe injection method, and the fragment ions generated by McLafferty rearrangement were still observed. Moreover, protonated $t$-Boc-MP could be detected.

Conclusions Hence, DART-TOF-MS provides a rapid and accurate method for the detection of $t$-Boc-MP, allowing suppression of the pyrolysis reaction and identification of both fragment ions and protonated $t$-Boc-MP. To our knowledge, this is the first report for detecting $t$-Boc-MP by MS techniques.
\end{abstract}

Keywords tert-Butoxycarbonyl-methamphetamine $(t$-Boc-MP) · "Masked" methamphetamine · Rapid detection by DARTTOF-MS $\cdot$ McLafferty rearrangement $\cdot$ Pyrolysis

\section{Introduction}

In Japan, methamphetamine (MP), amphetamine, and their salts are strictly regulated by the Stimulants Control Law, and represent the majority of drug-related arrests. MP is

Ken-ichi Sugie

sk00364@mhlw.go.jp

1 Narcotics Control Department, Kanto-Shin'etsu Regional Bureau of Health and Welfare Ministry of Health, Labour and Welfare, 1-2-1 Kudan-Minami, Chiyoda-ku, Tokyo 102-8309, Japan

2 Narcotics Control Department, Tohoku Regional Bureau of Health and Welfare Ministry of Health, Labour and Welfare, 3-2-23 Honchou, Aoba, Sendai 980-0014, Japan

3 Department of Analytical Chemistry, Faculty of Pharmaceutical Sciences, Hoshi University, 2-4-41 Ebara, Shinagawa-ku, Tokyo 142-8501, Japan seized in various forms such as crystal, tablet, and powder forms, and some MP seizures have been found to contain adulterants such as sodium benzoate and sodium thiosulfate [1]. In particular, $N$-isopropylbenzylamine, which is a structural isomer of MP [2], is one of the most common adulterants. In addition, the use of masked illegal substances as a method of drug smuggling is of increasing concern. In 2004, the Australian Custom Police seized methyl-3[3',4'-(methylene dioxy)phenyl]-2-methyl glycidate, which upon hydrolysis and decarboxylation efficiently produced 3,4-methylenedioxyphenyl-2-propanone, a precursor of 3,4-methylenedioxymethamphetamine (MDMA) [3].

Recently, phenethylamines bearing a tert-butoxycarbonyl ( $t$ Boc) group on the amine were seized [4-6]. The $t$-Boc group is one of the most commonly used amino-protecting groups in organic synthesis, such as peptide synthesis, because of the simple protection-deprotection procedures [7]. In general, the $t$-Boc group can be removed by treatment with a strong acid, 
such as trifluoroacetic acid, to give the original compound in high yield [8]. Deprotection can also be efficiently achieved by dissolution in distilled water at $100{ }^{\circ} \mathrm{C}$ within several tens of minutes, without using a strong acid [9]. In particular, seizures of $t$-Boc-protected MDMA and MP, phenethylamines containing secondary amino groups, have been reported. Namely, $t$-Boc-MDMA was found in a viscous and bright red liquid hair product by the Australian Custom Police. The substance was initially believed to be the MDMA precursor safrole, but further detailed analysis identified it as $t$-Boc-MDMA [4]. Moreover, $t$-Boc-MP mixed with a liquid detergent was seized in New Zealand [6]. In view of the simple protection-deprotection procedures, a variety of phenethylamine drugs could be easily masked by $t$-Boc protection of the amino group. However, no analytical data for $t$-Boc-protected phenethylamines is available to date, making drug detection difficult and inaccurate. Moreover, $t$-Boc-MP and $t$-Boc-MDMA are unregulated. Clearly, the objective of the amine-masking strategy would be to reduce the risk of smuggling illicit drugs by converting them into unregulated substances. Hence, in order to prevent their distribution worldwide, a rapid and accurate analytical method for the detection of $t$-Boc-masked drugs is needed to quickly provide the administrative authorities with the drug data.

Liquid chromatography-mass spectrometry (LC-MS) and gas chromatography-MS (GC-MS) are the most widely used techniques for illegal drug analysis. LC-timeof-flight-MS (LC-TOF-MS) is particularly useful for identifying unknown compounds, because it can easily detect protonated molecules and accurately measure the molecular mass [10,11]. However, analytical techniques based on chromatographic separations, such as LC-TOF-MS and GC-MS, usually require relatively long measurement times. On the other hand, MS using a direct analysis in real time (DART) ion source developed by Cody et al. [12] is a rapid analytical method as compared to GC-MS and LC-TOFMS. Moreover, high-resolution TOF-MS allows the estimation of chemical formulas from accurate mass data. Thus, DART-TOF-MS has been widely used as a screening technique in the field of food hygiene and forensic science [13, 14]. On the basis of these considerations, we envisioned that DART-TOF-MS would provide an efficient screening method for $t$-Boc-MP.

In this study, a rapid and accurate DART-TOF-MS screening method for $t$-Boc-MP was developed. The efficiency of the method was evaluated by comparison with GC-MS and LC-TOF-MS analyses.

\section{Materials and methods}

\section{Materials}

MP hydrochloride, which was provided by the Ministry of Health, Labor and Welfare (Tokyo, Japan), was used in this study. Di-tert-butyl dicarbonate was purchased from Tokyo Chemical Industry Co., Ltd. (Tokyo, Japan). All other reagents were of special grade from Kanto Kagaku Co., Ltd. (Tokyo, Japan). $t$-Boc-MP was synthesized according to the procedure described by Davis et al. [8] for the preparation of $t$-Boc-amine. The purity of synthesized $t$-Boc-MP was confirmed using GC-flame ionization detection ( $\geqq 98 \%)$. The microsyringe used (capacity $10 \mu \mathrm{L}, 23-26$-gauge needle) was purchased from Hamilton Inc. (Reno, NV, USA).

\section{GC-MS conditions}

\section{GC-MS instrument}

Agilent 7890A GC/5975C MSD system (Agilent Technologies, Santa Clara, CA, USA); column: DB-5MS $(30 \times 0.25-$ $\mathrm{mm}$ i.d., film thickness $0.25 \mu \mathrm{m}$; Agilent Technologies); inlet temperature: $250^{\circ} \mathrm{C}$; oven temperature: 2 min at $60^{\circ} \mathrm{C}, 10^{\circ} \mathrm{C} /$ min to $300{ }^{\circ} \mathrm{C}$, and $5 \mathrm{~min}$ at $300{ }^{\circ} \mathrm{C}$; transfer line temperature: $280^{\circ} \mathrm{C}$; injection volume: $1 \mu \mathrm{L}$; injection mode: split (20:1) or splitless; carrier gas: $\mathrm{He}(1.2 \mathrm{~mL} / \mathrm{min})$; ionization conditions: electron ionization, $70 \mathrm{eV}, 150{ }^{\circ} \mathrm{C}$; mass range: $\mathrm{m} / \mathrm{z} 40-500$.

\section{LC-TOF-MS conditions}

LC-TOF-MS was used in MS ${ }^{\mathrm{E}}$ mode, which allowed alternating low and high collision energies in one injection, thus providing precursor and fragment/product ion information, respectively. The following operational conditions were used.

\section{LC instrument}

ACQUITY UPLC instrument (Waters, Milford, MA, USA); column: ACQUITY UPLC HSS C18 column $(150 \times 2.1$-mm i.d., particle size $1.8 \mu \mathrm{m}$; Waters); solvent A: $5 \mathrm{mM}$ ammonium formate in water, $\mathrm{pH} 3$; solvent $\mathrm{B}$ : $0.1 \%$ formic acid in acetonitrile; flow rate: $0.4 \mathrm{~mL} / \mathrm{min}$; elution program: $80 \%$ A/20\% B (2-min hold) to $20 \% \mathrm{~A} / 80 \% \mathrm{~B}$ (2-15 min, 8-min hold); injection volume: $1 \mu \mathrm{L}$; column temperature: $50^{\circ} \mathrm{C}$.

\section{TOF-MS instrument}

Xevo G2 QToF mass spectrometer (Waters); ion source: electrospray ionization in positive mode; ion source temperature: $150{ }^{\circ} \mathrm{C}$; capillary and cone voltages: 830 and $40 \mathrm{~V}$, respectively; collision energy function 1: $6 \mathrm{~V}$; collision energy 
function 2: 10-40 V; mass range: $m / z, 50-1000$; scan time: $0.2 \mathrm{~s}$; lock mass: leucine enkephalin $(\mathrm{m} / \mathrm{z}, 556.2771)$.

\section{DART-TOF-MS conditions}

\section{DART ion source instrument}

DART-SVPTM (Ionsense, Saugus, MA, USA); ionization mode: positive mode; helium gas flow: $3.5 \mathrm{~L} / \mathrm{min}$; ion source temperature: $200{ }^{\circ} \mathrm{C}$; discharge electrode needle voltage: $3200 \mathrm{~V}$

\section{Mass spectrometer}

JMS-100LP AccuTOF LC-Plus (JEOL, Tokyo, Japan); orifice 1 voltage: $10 \mathrm{~V}$; orifice 2 voltage: $5 \mathrm{~V}$; orifice 1 temperature: $180{ }^{\circ} \mathrm{C}$; ring lens voltage: $5 \mathrm{~V}$; ion guide peak voltage: $300 \mathrm{~V}$; reflectron voltage: $980 \mathrm{~V}$; mass range: $\mathrm{m} / \mathrm{z}$ 10-1000; internal mass number calibration: polyethylene glycol 200 and 400; sample injection method: microsyringe.

\section{Results and discussion}

\section{GC-MS}

\section{Structural confirmation of the synthesized $t$-Boc-MP}

The synthesized $t$-Boc-MP was analyzed by GC-MS, and the obtained mass spectrum was compared to that of $t$-BocMP reported by Collins et al. [4]. A solution of $t$-Boc-MP in methanol was prepared at a concentration of $100 \mu \mathrm{g} / \mathrm{mL}$, and the sample was analyzed by GC-MS, using a split ratio of 20:1 and an inlet temperature at $250{ }^{\circ} \mathrm{C}$. Notably, the mass spectrum of the synthesized $t$-Boc-MP (Fig. 1) was identical to the published one [4].

\section{Pyrolysis of $t$-Boc-MP in the GC-MS injection port}

MP was detected in the total ion current chromatogram (TICC) by GC-MS for the synthesized $t$-Boc-MP, and was presumably generated by pyrolysis of $t$-Boc-MP in the injection port of the instrument. In order to investigate the pyrolysis behavior of $t$-Boc-MP, GC-MS analyses were carried out at different inlet temperatures, namely, $200,220,250$, and $300{ }^{\circ} \mathrm{C}$, and at a split ratio of $20: 1$. The chromatogram recorded at an inlet temperature of $300{ }^{\circ} \mathrm{C}$ is shown in Fig. 2, and the MP-to-t-Boc-MP peak area ratios and $t$-Boc-MP peak areas at each inlet temperature are summarized in Table 1. MP was detected at 250 and $300{ }^{\circ} \mathrm{C}$, but not at 200 and $220{ }^{\circ} \mathrm{C}$. Notably, the MP-to- $t$-Boc-MP peak area ratio increased with increasing inlet temperature, whereas the $t$-Boc-MP peak area decreased. Next, the effect

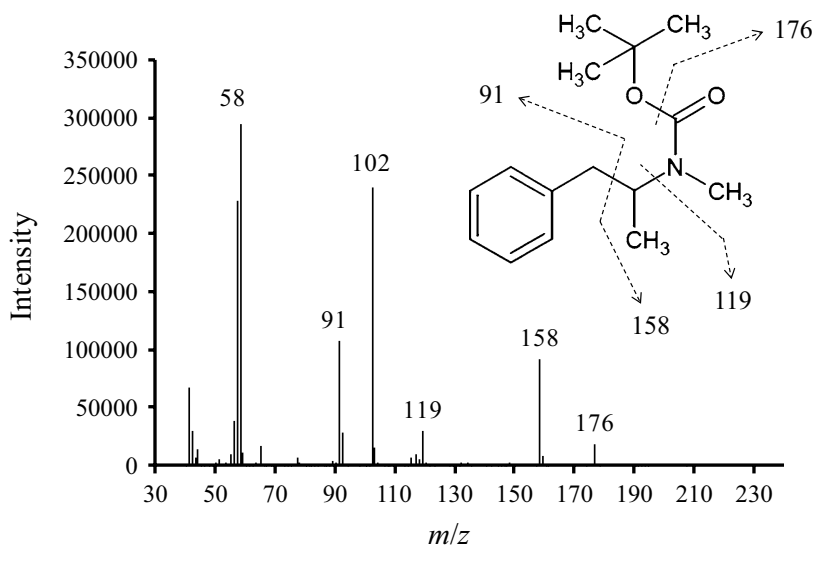

Fig. 1 Mass spectrum of tert-butoxycarbonyl-methamphetamine ( $t$-Boc-MP) measured by gas chromatography-mass spectrometry (GC-MS) with assigned fragmentation pattern using a split ratio of 20:1 and inlet temperature at $250{ }^{\circ} \mathrm{C}$

of the injection mode on the pyrolysis of $t$-Boc-MP was investigated, and the results are summarized in Table 2. The injector was operated in splitless and split modes (namely, $10: 1,20: 1$, and 50:1), while the inlet temperature was set to $200{ }^{\circ} \mathrm{C}$. MP was detected when the GC was operated at a 10:1 split ratio and in splitless mode, but not at split ratios of 50:1 and 20:1. In splitless injection, the $t$-Boc-MP peak area was, of course, larger than that obtained using a split injection mode, suggesting that the pyrolysis of $t$-Boc-MP in the injection port was promoted at lower split ratios, which are associated with a longer heating time. These results confirmed that MP was generated by pyrolysis of $t$-Boc-MP in the injection port during GC-MS analysis. Hence, specific GC-MS conditions can lead to the formation of MP with the risk of incorrect analytical results.

\section{LC-TOF-MS}

A $100-\mathrm{ng} / \mathrm{mL}$ methanol solution of $t$-Boc-MP was prepared and analyzed by LC-TOF-MS. As can be seen from the mass spectrum in Fig. 3, the ion at $m / z 91.054$ was detected as the base peak, and fragment ion peaks at $m / z 119.085$, 150.127 , and 194.117 were also observed. However, protonated $t$-Boc-MP (theoretical $m / z$ value: 250.180 ) was not detected. According to the report of Wolf et al. [15], $t$-Bocprotected amines undergo McLafferty rearrangement in the ion source of the LC-MS instrument. Thus, a similar rearrangement of $t$-Boc-MP was expected to occur during LC-TOF-MS analysis, and the two previously described pathways [15] were considered. In one pathway, isobutene desorption generates an intermediate $(\mathrm{m} / \mathrm{z}$ 194.117), which undergoes decarboxylation to produce MP $(\mathrm{m} / \mathrm{z}, 150.127$; Fig. 4a). In the second pathway, isobutene desorption and 

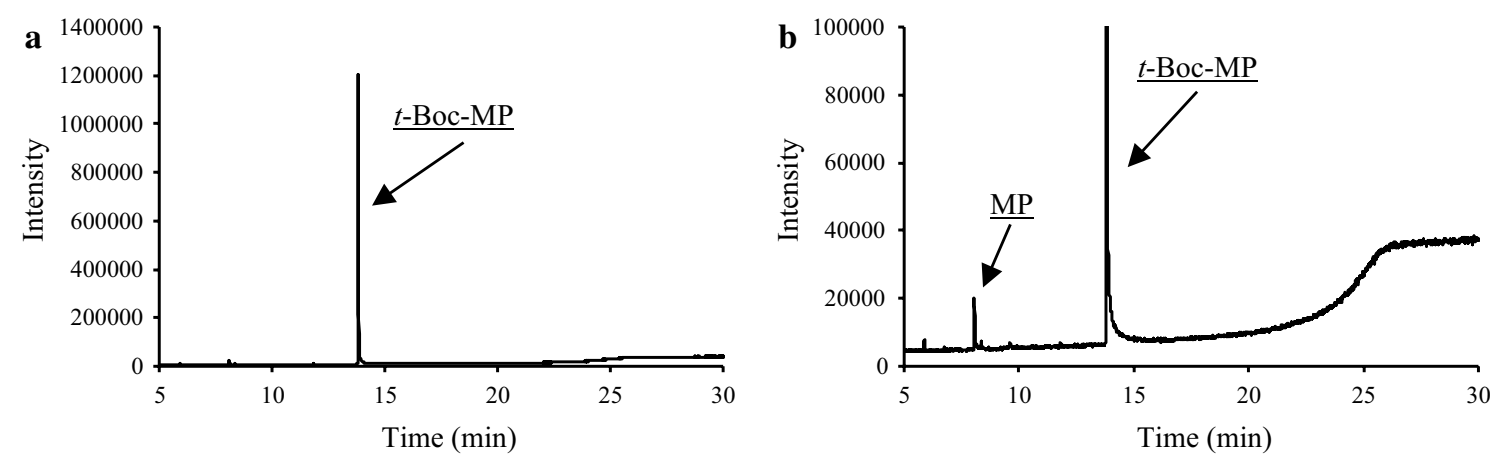

Fig. 2 GC-MS total ion current chromatograms (TICCs) of $t$-Boc-MP at an injector temperature of $300{ }^{\circ} \mathrm{C}$. b Expanded view of the chromatogram shown in $\mathbf{a}$

Table 1 Methamphetamine (MP)-to-tert-butoxycarbonyl-methamphetamine $(t$-Boc-MP) peak area ratios and $t$-Boc-MP peak areas measured by gas chromatography-mass spectrometry (GC-MS) at different inlet temperatures with split injection at 20:1

\begin{tabular}{lll}
\hline $\begin{array}{l}\text { Inlet tempera- } \\
\text { ture }\left({ }^{\circ} \mathrm{C}\right)\end{array}$ & $\begin{array}{l}\text { Peak area ratio (MP-to- } \\
t \text {-Boc-MP; } \pm \mathrm{SD}, n=3)\end{array}$ & $\begin{array}{l}t \text {-Boc-MP area } \\
\left(\times 10^{7}, \pm \mathrm{SD}, n=3\right)\end{array}$ \\
\hline 200 & 0 & $3.7 \pm 0.2$ \\
220 & 0 & $3.0 \pm 0.1$ \\
250 & $0.0022 \pm 0.000049$ & $2.6 \pm 0.3$ \\
300 & $0.0145 \pm 0.00061$ & $2.3 \pm 0.7$ \\
\hline
\end{tabular}

$S D$ standard deviation

Table 2 MP-to- $t$-Boc-MP peak area ratios and $t$-Boc-MP peak areas measured by GC-MS for each injection mode at inlet temperature of $200{ }^{\circ} \mathrm{C}$

\begin{tabular}{lll}
\hline Injection mode & $\begin{array}{l}\text { Peak area ratio (MP-to- } \\
t \text {-Boc-MP; } \pm \mathrm{SD}, n=3)\end{array}$ & $\begin{array}{l}t \text {-Boc-MP area } \\
\left(\times 10^{7}, \pm \mathrm{SD}, n=3\right)\end{array}$ \\
\hline Splitless & $0.012 \pm 0.00034$ & $80.2 \pm 0.06$ \\
Split $(10: 1)$ & $0.0024 \pm 0.00017$ & $5.27 \pm 0.01$ \\
Split $(20: 1)$ & 0 & $3.70 \pm 0.21$ \\
Split (50:1) & 0 & $0.67 \pm 0.02$ \\
\hline
\end{tabular}

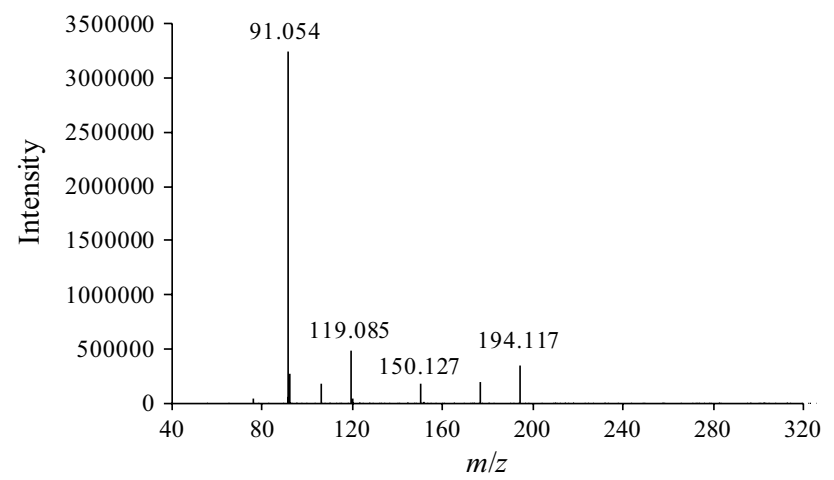

Fig. 3 Mass spectrum of $t$-Boc-MP measured by liquid chromatography-time-of-flight-mass spectrometry (LC-TOF-MS) decarboxylation occur simultaneously to yield MP directly (Fig. 4b). In our study, both fragment ions at $\mathrm{m} / \mathrm{z} 150.127$ and 194.117 were detected, indicating that the McLafferty rearrangement of $t$-Boc-MP proceeded via the pathway shown in Fig. 4a. Hence, although it was difficult to detect the protonated molecular ion of $t$-Boc-MP by LC-TOF-MS, detection of the fragment ions derived from the McLafferty rearrangement allows the identification of $t$-Boc-MP.

\section{DART-TOF-MS}

\section{Comparison of sample injection methods}

A glass rod is commonly used as a sample injection device for DART-TOF-MS; however, the sample peak shape degraded into split peaks in the chromatogram. In order to overcome this problem, a microsyringe injection method was previously devised and applied to the quantitative analysis of new psychoactive substances including $\alpha$-pyrrolidinovalerophenone by DART-TOF-MS [16]. Thus, the effectiveness of the microsyringe injection method for DART-TOF-MS analysis of $t$-Boc-MP was examined in this study. A $100-\mu \mathrm{g} / \mathrm{mL}$ methanol solution of $t$-Boc-MP was analyzed by DART-TOF-MS at an ion source temperature of $250{ }^{\circ} \mathrm{C}$ using a glass rod or a microsyringe as a sample injection method [16]. The TICCs and extracted ion chromatograms of protonated $t$-Boc-MP $(m / z$ 250.180) obtained from the corresponding TICC are shown in Fig. 5. Using the microsyringe injection technique, distinct peaks could be identified in TICC (Fig. 5a), which were confirmed by sharp peaks in the extracted ion chromatogram (Fig. 5c). On the contrary, using a glass rod for sample injection, individual peaks were not resolved in TICC (Fig. 5b), but could be observed in the corresponding extracted ion chromatogram (Fig. 5d). However, deterioration of the peak shape was observed due to tailing (Fig. 5d). These results suggest that the microsyringe injection method was more suitable for sensitive detection of $t$-Boc-MP by DART-TOF-MS. 

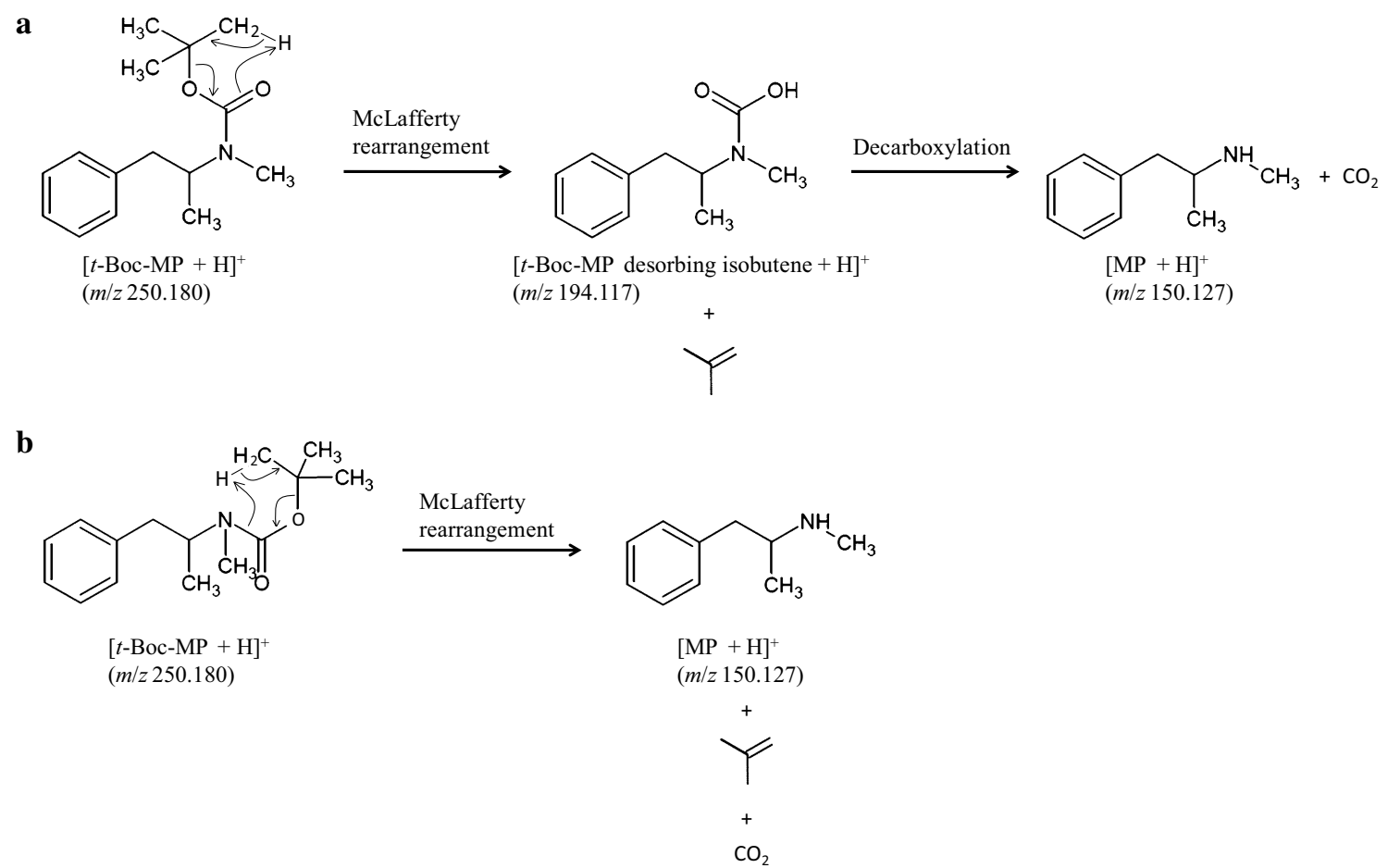

Fig. 4 Possible pathways for the McLafferty rearrangement of $t$-BocMP during LC-TOF-MS analysis: a pathway producing an intermediate derived from isobutene desorption $(\mathrm{m} / \mathrm{z}, 194.117)$ and metham-

\section{Effect of solvent}

In order to determine the best solvent for DART-TOF-MS analysis, $100-\mu \mathrm{g} / \mathrm{mL} t$-Boc-MP solutions were prepared in different solvents, namely, methanol, ethanol, isopropanol, acetonitrile, and ethyl acetate. All samples were analyzed at an ion source temperature of $200{ }^{\circ} \mathrm{C}$. Notably, protonated $t$-Boc-MP $(\mathrm{m} / \mathrm{z}, 250.180)$ was detected in all solutions (Fig. 6), whereas protonated MP $(\mathrm{m} / \mathrm{z}, 150.128)$ was detected in all solvents except for the ethyl acetate solution. Moreover, the protonated isobutene-desorbed $t$-Boc-MP $(\mathrm{m} / z$ 194.118; Fig. 4a) was found in all solutions. This suggests that $t$-Boc-MP underwent the McLafferty rearrangement also during DART-TOF-MS analysis. Moreover, unlike the case of GC-MS, pyrolysis was not observed in the DART-TOF-MS measurements, irrespective of the solvent used.

In addition, all spectra showed a peak at $m / z 499.352$, which was attributed to the dimer of $t$-Boc-MP, whereas the peaks at $\mathrm{m} / z 399.300$ and 443.003 , corresponding to the dimers of $t$-Boc-MP with MP and with isobutene-desorbed $t$-Boc-MP, respectively, were detected in all spectra except for the ethyl acetate sample. However, in the case of the ethyl acetate sample, the most intense peak was found at $m / z$ 177.122, which corresponded to the dimer form of phetamine (MP) $(\mathrm{m} / \mathrm{z}, 150.127)$; b pathway producing only MP $(\mathrm{m} / \mathrm{z}$ 150.127). All $\mathrm{m} / z$ values refer to the corresponding protonated compounds

ethyl acetate. Moreover, the impurity peaks related to the solvent and water molecules were detected in the mass range below $m / z 150.128$ in all solvent samples. The impurity peaks related to methanol had lower ionic strengths as compared to those for the other solvents. Hence, in this respect, methanol proved to be the best solvent for DART-TOF-MS analysis of $t$-Boc-MP.

\section{Effect of ion source temperature}

The influence of the DART ion source temperature was investigated by analyzing the $t$-Boc-MP methanol solution at 200,250 , and $300{ }^{\circ} \mathrm{C}$. As a result, the peak of protonated $t$-Boc-MP was detected also at ion source temperatures of 250 and $300{ }^{\circ} \mathrm{C}$, with lower intensity as compared to that of the spectrum at $200{ }^{\circ} \mathrm{C}$. On the other hand, protonated dimers were almost not detected at an ion source temperature of $300{ }^{\circ} \mathrm{C}$ (Fig. 7). Moreover, the intensity of the MP ion $(\mathrm{m} / \mathrm{z}, 150.128)$ generated by McLafferty rearrangement increased with increasing ion source temperature (Figs. 6a, 7b). According to Harris et al. [17], the internal energy of analytes increases with increasing DART ion source temperature. This increase in internal energy can explain the increased formation of MP by McLafferty rearrangement and the lower formation of $t$-Boc-MP and dimeric species. 
a $\operatorname{TICC}$ (microsyringe injection)

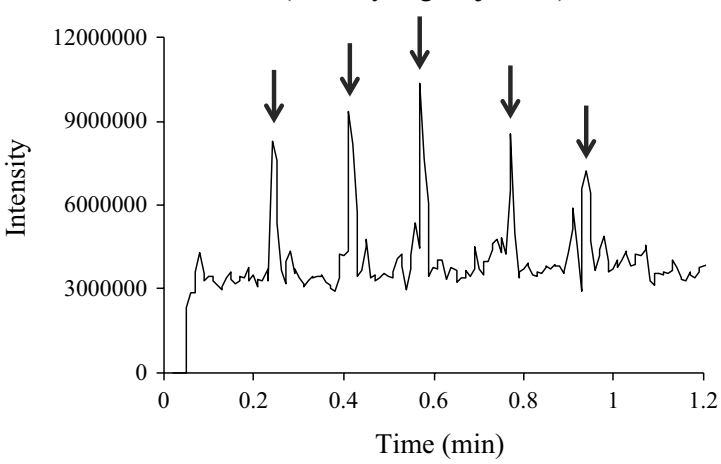

c Extracted ion chromatogram

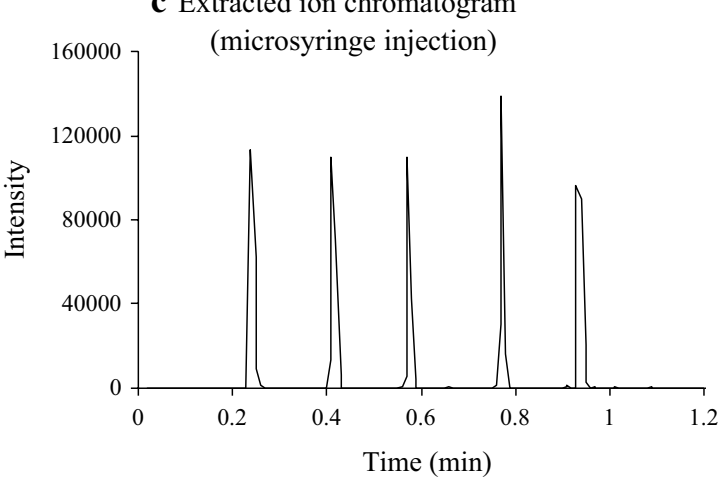

Fig. 5 Comparison between microsyringe and glass rod injection methods in the direct analysis in real time-time-of-flight-mass spectrometry (DART-TOF-MS) analysis of $t$-Boc-MP $(100 \mu \mathrm{g} / \mathrm{mL}$ in methanol): a TICC obtained using a microsyringe, b TICC obtained

Hence, an ion source temperature at $200{ }^{\circ} \mathrm{C}$ was more suitable for reliable detection of protonated $t$-Boc-MP and fragment ions by DART-TOF-MS analysis.

\section{Effect of sample concentration}

Accurate identification of $t$-Boc-MP by DART-TOF-MS analysis could be achieved by the detection of not only protonated $t$-Boc-MP but also the fragment ions generated from the McLafferty rearrangement, which could be improved by suppressing the protonated dimer peaks. Because the dimers were formed at a high $t$-Boc-MP concentration of $100 \mu \mathrm{g} / \mathrm{mL}$, the effect of sample concentration on the DART-TOF-MS measurement was examined by analyzing a low concentration $(10-\mu \mathrm{g} / \mathrm{mL})$ of $t$-BocMP in methanol. Notably, the dimeric species were not detected at ion source temperatures of 200, 250, and $300{ }^{\circ} \mathrm{C}$; however, the intensity of the protonated $t$-Boc-MP ion peak decreased at ion source temperatures of 250 and $300{ }^{\circ} \mathrm{C}$ (Fig. 8). Moreover, using an ion source temperature of $200{ }^{\circ} \mathrm{C}$, the intensity of $\mathrm{m} / \mathrm{z}, 150.128$ was higher at a concentration of $10 \mu \mathrm{g} / \mathrm{mL}$ than at $100 \mu \mathrm{g} / \mathrm{mL}$ (Fig. 6a), and a relatively high intensity of the protonated $t$-Boc-MP b TICC (glass rod injection)

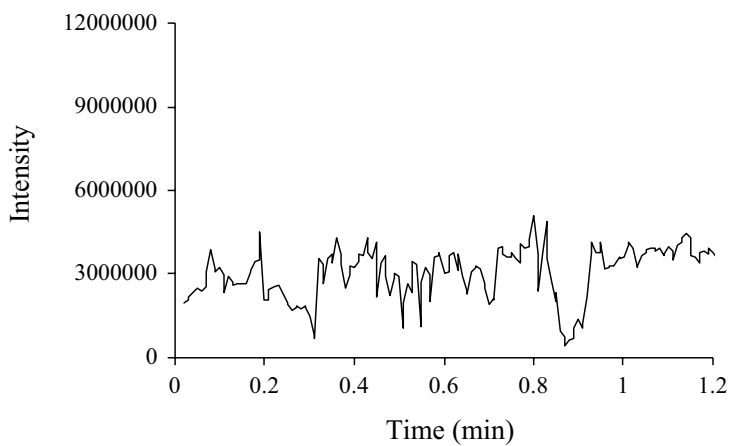

d Extracted ion chromatogram (glass rod injection)

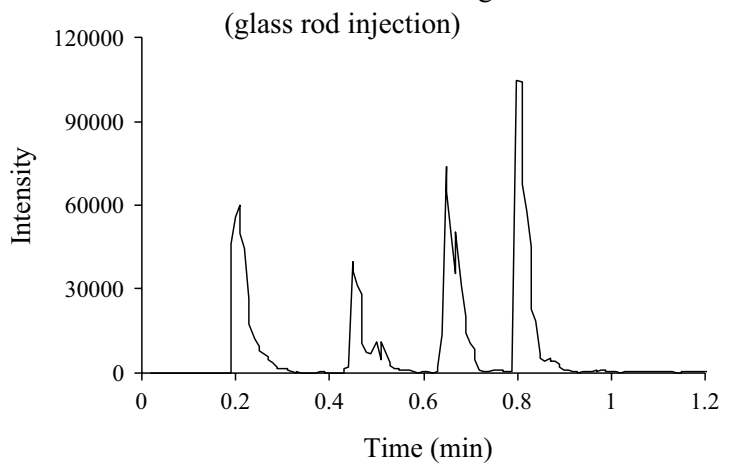

using a glass rod, c extracted ion chromatogram $(\mathrm{m} / \mathrm{z}$ 250.180) obtained from a, d extracted ion chromatogram $(\mathrm{m} / \mathrm{z}, 250.180)$ obtained from $\mathbf{b}$. The black arrows in a indicate the peaks of $t$-BocMP

peak was observed. These results show that the formation of dimers can be suppressed by using a diluted sample, which allows highly sensitive detection of fragment ions and protonated $t$-Boc-MP by DART-TOF-MS.

\section{Conclusions}

In this study, a DART-TOF-MS method was developed for the rapid and accurate detection of $t$-Boc-MP by using a microsyringe injection technique, methanol as a solvent, an ion source temperature of $200{ }^{\circ} \mathrm{C}$, and a sample concentration of $10 \mu \mathrm{g} / \mathrm{mL}$. This method, as a screening test, proved to be superior to GC-MS and LC-TOF-MS analyses. During GC-MS analysis, $t$-Boc-MP underwent pyrolysis in the injection port under certain operational conditions, which can lead to an analytical error. In the LC-TOF-MS spectrum, ion fragments generated by McLafferty rearrangement were detected, whereas the protonated $t$-BocMP parent ion was not found. On the other hand, in the DART-TOF-MS analysis, pyrolysis was not observed with any of the solvents. Moreover, similarly to LC-TOF-MS, in the DART-TOF-MS spectrum, the fragment ions formed by 

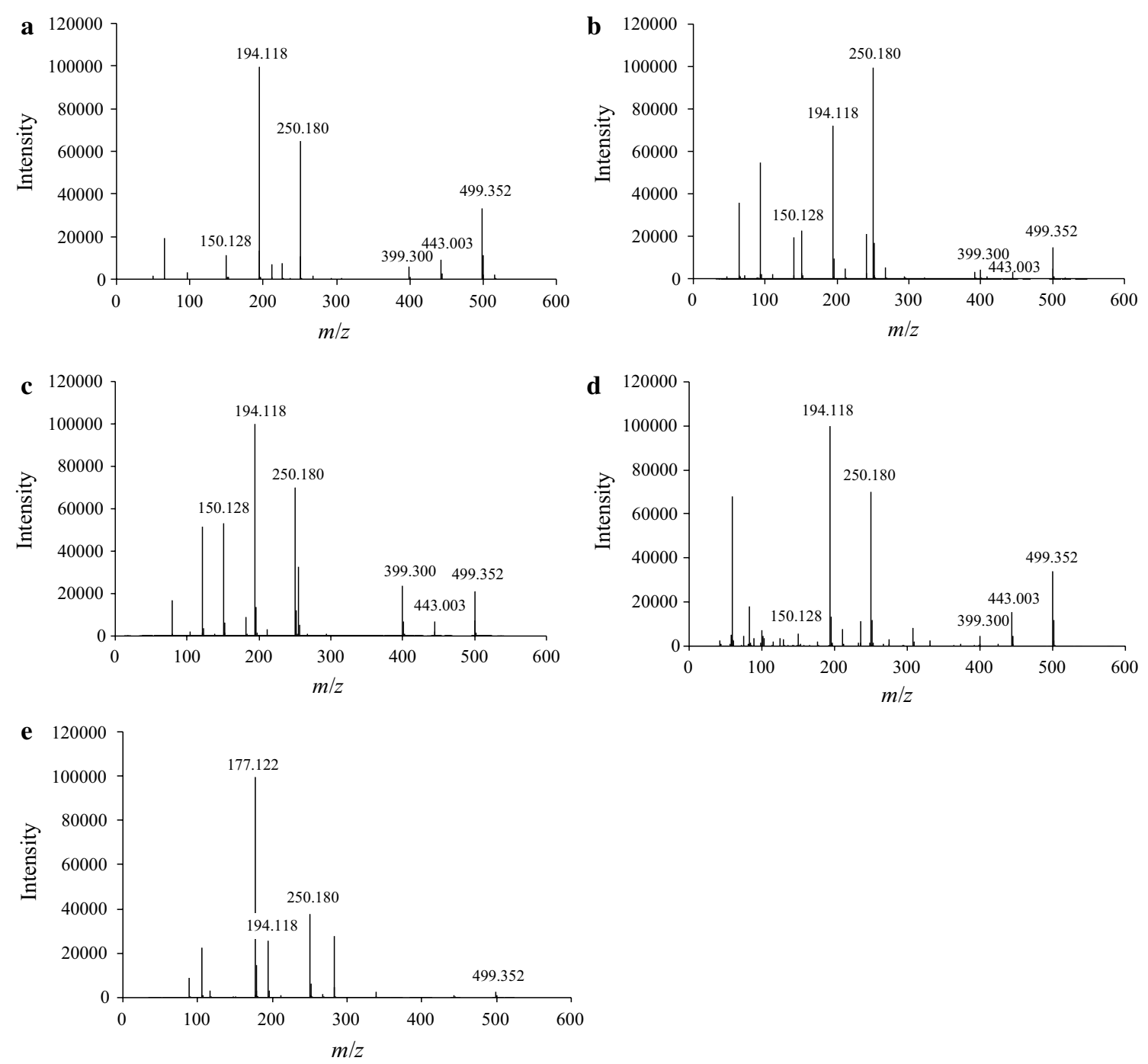

Fig. 6 Comparison of DART-TOF-MS spectra of $t$-Boc-MP $(100 \mu \mathrm{g} / \mathrm{mL})$ in different solvents: a methanol, b ethanol, $\mathbf{c}$ isopropanol, $\mathbf{d}$ acetonitrile, and e ethyl acetate solutions. The ion source temperature was set at $200{ }^{\circ} \mathrm{C}$
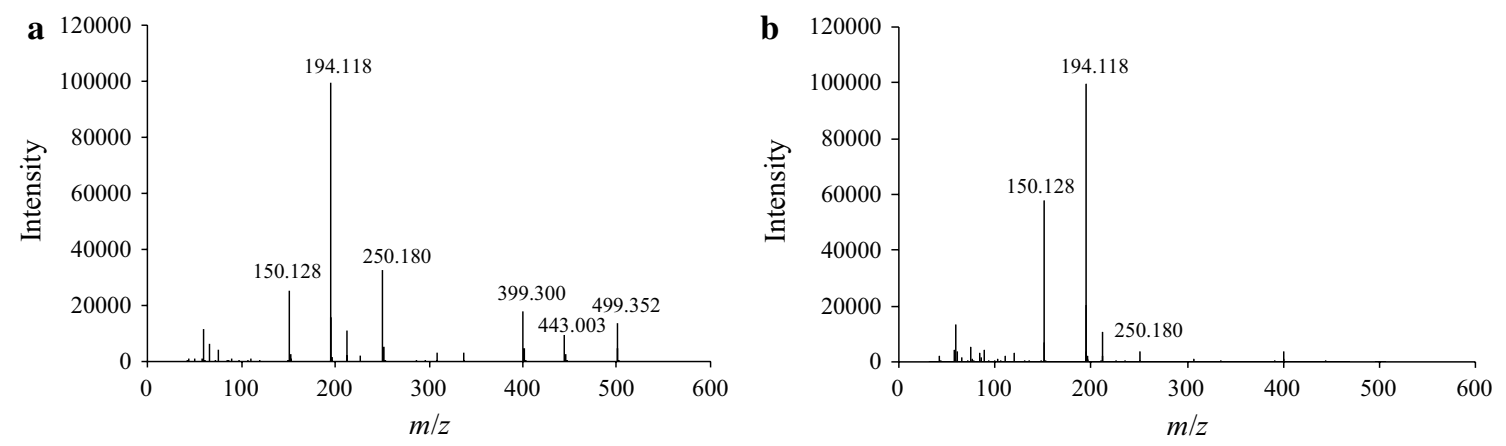

Fig. 7 DART-TOF-MS spectra of $t$-Boc-MP. DART ion source temperatures: a $250{ }^{\circ} \mathrm{C}, \mathbf{b} 300{ }^{\circ} \mathrm{C}$ 

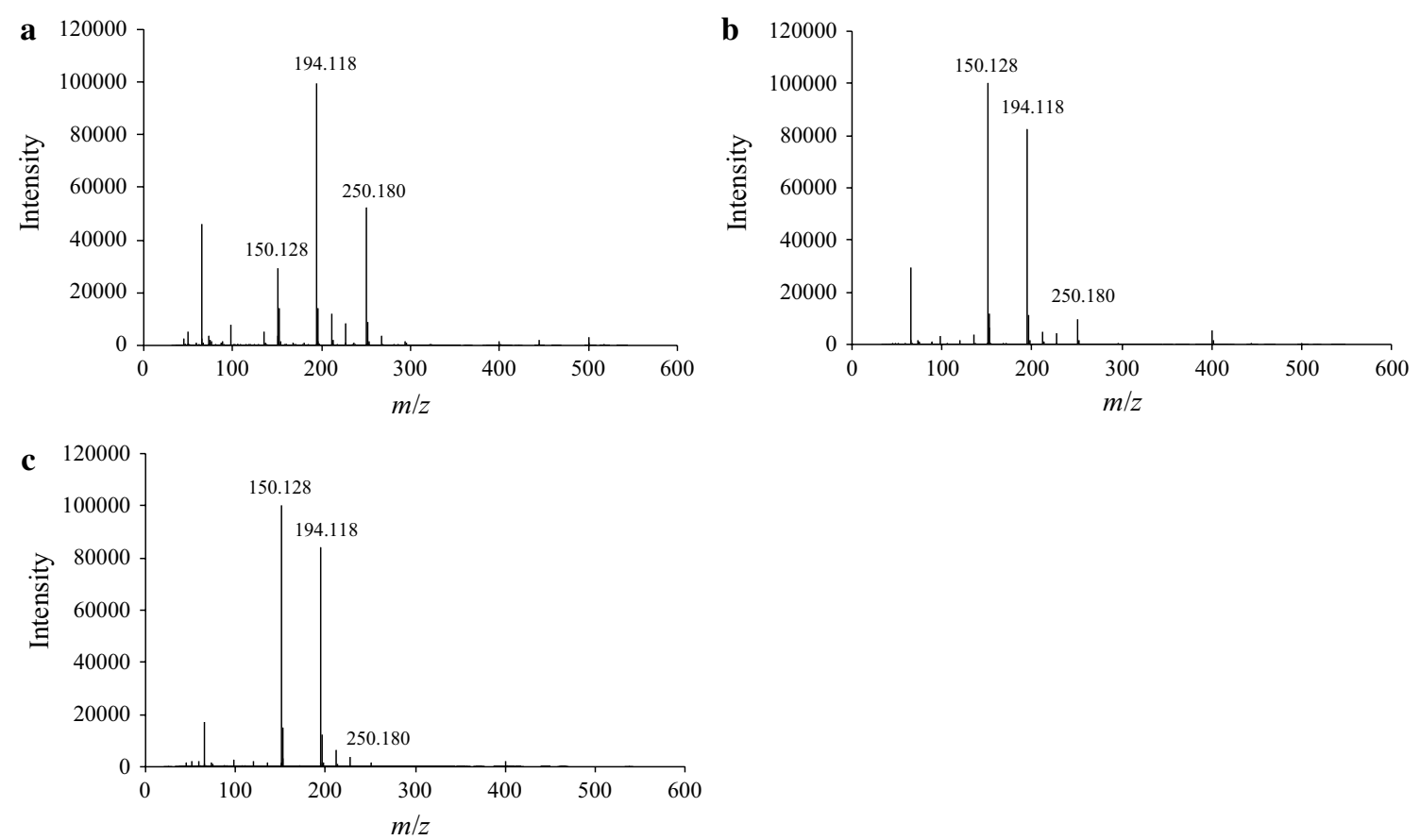

Fig. 8 DART-TOF-MS spectra of $t$-Boc-MP at a lower concentration $(10 \mu \mathrm{g} / \mathrm{mL})$. DART ion source temperatures: a 200 , b 250 , and $\mathbf{c} 300{ }^{\circ} \mathrm{C}$

McLafferty rearrangement were detected, as well as the protonated $t$-Boc-MP peak. Hence, DART-TOF-MS provides a rapid and accurate method for the detection of $t$-Boc-MP with significant reduction of the analytical error by suppression of the pyrolysis reaction, and for the identification not only with fragment ions but also with the protonated $t$-BocMP. To our knowledge, this is the first report dealing with detection of $t$-Boc-MP by MS techniques.

Acknowledgements A portion of this work was supported by a Health and Labour Sciences Research Grant from the Ministry of Health, Labour and Welfare, Japan.

\section{Compliance with ethical standards}

Conflict of interest There are no financial or other relationships that could lead to a conflict of interest.

Ethical approval This article does not contain any studies with human participants or animals performed by any of the authors.

Open Access This article is distributed under the terms of the Creative Commons Attribution 4.0 International License (http://creativecomm ons.org/licenses/by/4.0/), which permits unrestricted use, distribution, and reproduction in any medium, provided you give appropriate credit to the original author(s) and the source, provide a link to the Creative Commons license, and indicate if changes were made.

\section{References}

1. Inoue H, Iwata YT, Kuwayama K (2008) Characterization and profiling of methamphetamine seizures. J Health Sci 54:615-622

2. Sanderson RM (2008) Identification of N-methylbenzylamine hydrochloride, N-ethylbenzylamine hydrochloride, and $\mathrm{N}$-isopropylbenzylamine hydrochloride. Microgram J 6:36-45

3. Collins M, Heagney A, Cordaro F, Odgers D, Tarrant G, Stewart S (2007) Methyl 3-[3',4'-(methylenedioxy)phenyl]-2-methyl glycidate: an ecstasy precursor seized in Sydney, Australia. J Forensic Sci 52:898-903

4. Collins M, Donnelly C, Cameron S, Tahtouh M, Salouros H (2017) Identification and characterization of $N$-tert-butoxycarbonyl-MDMA: a new MDMA precursor. Drug Test Anal 9:399-404

5. Westphal F, Grreser U, Holz K, Erkens M (2016) Strukturaufkärung und analytische Daten eines ungewöhnlichen MDMA-Derivates (in German). Toxichem Krimtech 83:92-102

6. Herald NZ (2017) Masked meth: first time method used, as four men charged with importing $\$ 100 \mathrm{~m}$ of the substance. http://www. Nzherald.co.nz/nz/news/article.cfm?c_id=1\&objectid=11818866 . Accessed 15 Mar 2017

7. Houghten RA (1985) General method for the rapid solid-phase synthesis of large number of peptide: specificity of antigen-antibody interaction at the level of individual amino acids. Proc Natl Acad Sci USA 82:5131-5135

8. Davis FA, Yang B, Deng J (2003) Asymmetric synthesis of cis5-tert-butylproline with metal carbenoid $\mathrm{NH}$ insertion. J Org Chem 68:5147-5152

9. Zinelaabidine C, Souad O, Zoubir J, Malika B, Nour-Eddine A (2012) A simple and efficient green method for the deprotection of $N$-Boc in various structurally diverse amines under water-mediated catalyst-free conditions. Int J Chem 4(3):73-79

10. Shintani-Ishida K, Nakamura M, Tojo M, Idota N, Ikegaya $\mathrm{H}$ (2015) Identification and quantification of 
4'-methoxy- $\alpha$-pyrrolidinobutiophenone (4-MeOPBP) in human plasma and urine using LC-TOF-MS in an autopsy case. Forensic Toxicol 33:348-354

11. Hernandez F, Ibenez M, Botero-Coy A-M, Bade R, BustosLopez MC, Rincon J, Moncavo A, Bijlsma L (2015) LC-QTOFMS screening of more than 1,000 licit and illicit drugs and their metabolites in wastewater and surface waters from the area of Bogotá, Colombia. Anal Bioanal Chem 21:6405-6416

12. Cody RB, Laramée JA, Durst DH (2005) Versatile new ion source for the analysis of materials in open air under ambient conditions. Anal Chem 77:2297-2302

13. Lesiak AD, Adams KJ, Domin MA, Henck C, Shepard JRE (2014) DART-MS for rapid, preliminary screening of urine for DMAA. Drug Test Anal 6:788-796

14. Martinez-Villalba A, Vaclavik L, Moyano E, Calceran MT, Hajslove J (2013) Direct analysis in real time high-resolution mass spectrometry for high-throughput analysis of antiparasitic veterinary drugs in feed and food. Rapid Commun Mass Spectrom 27:467-475

15. Wolf C, Villalobos CN, Cummings PG, Kennedy-Gabb S, Olsen MA, Trescher G (2005) Elucidation of the presence and location of $t$-Boc protecting groups in amines and dipeptides using oncolumn H/D exchange HPLC/ESI/MS. J Am Soc Mass Spectrom 16:553-564

16. Sugie K, Akutsu M, Saito K (2016) Quantitative analysis of NPS (new psychoactive substance) containing $\alpha$-PVP by direct analysis in real time (DART)-TOF-MS with micro syringe injection (in Japanese with English abstract). Bunseki Kagaku 65:439-446

17. Harris GA, Hostetler DM, Hampton CY, Fernández FM (2010) Comparison of the internal energy deposition of direct analysis in real time and electrospray ionization time-of-flight mass spectrometry. J Am Soc Mass Spectrom 21:855-863 